Please do not remove this page

RMIT

UNIVERSITY

\title{
Visualisation and auralisation of architectural design in a game engine based collaborative virtual environment
}

Harvey, Lawrence; Moloney, Jules

https://researchrepository.rmit.edu.au/esploro/outputs/9921858069401341/filesAndLinks?institution=61RMIT_INST\&index=null

Harvey, L., \& Moloney, J. (2004). Visualisation and auralisation of architectural design in a game engine based collaborative virtual environment. Information Visualisation, 8th International Conference on (IV'04) Proceedings Online, 827-832. https://doi.org/10.1109/IV.2004.1320236

Published Version: https://doi.org/10.1109/IV.2004.1320236

Repository homepage: https://researchrepository.rmit.edu.au

(C) 2004 IEEE

Downloaded On 2023/04/26 14:24:44 +1000

Please do not remove this page 


\title{
Visualization and 'Auralization' of Architectural Design in a Game Engine Based Collaborative Virtual Environment
}

\author{
Jules Moloney and Lawrence Harvey \\ University of Auckland and the Spatial InformationArchitecture Laboratory, RMIT University \\ j.moloney@auckland.ac.nz, lawrence.harvey@sial.edu.au
}

\begin{abstract}
This paper describes the development of collaborative virtual environment (CVE) software based on a game engine to support the early stages of design in the context of architectural education. The precedent of narrative drawing technique in architecture is introduced. From this we position the use of a CVE as a means to explore design context in a manner that is not possible when using typical architectural visualization software. Outcomes from software trials are reported that establish the value of working in a real time environment where design iterations can be tested from multiple points of view, and the important role of $3 D$ sound to evoke occupancy and materiality. Negative results are reported in relation to collaborative design functionality and a revised approach based on an integrated project database is described.
\end{abstract}

\section{Introduction:}

" Because architecture and product design is still executed through the medium of two dimensional drawing (or its electronic equivalent) the 'objective' properties of the product or place tend to be stressed at the expense of subjective aspects, including sensual qualities, such as light, heat, sound, humidity and so on which cannot easily quantified or specified" [1].

John Thackara's reference above to drawing in his description of the design primario theories of the late 1980 's is posited in relation to $2 \mathrm{D}$ visualization practices prevalent in design studios at that time. Two dimensional drawing practices were perceived as reductive, analytical and incapable of communicating the subjective aspects of design. Today the proliferation of 3D computer aided design has transformed the means of design representation with sophisticated visualization of form and surface now well established within education and the profession. However the emphasis has still being on the visual appearance of the designed object and in this sense continues the trajectory of the architectural illustrator the emphasis is on appearance usually from a particular viewing position that shows the design to best advantage. Thackara's essay was titled "Beyond the Object in Design" and it is in that spirit that we have established a research program in which one aim is to broaden the manner in which architectural design has been historically conceived as projective geometry that, more often than not, has been devoid of physical or temporal context. This broadening is based on the use of virtual environments that allow some exploration of the subjective agenda of design visualization: the use of real-time graphics to explore the temporal dimensions of context; spatialized sound to evoke qualities of space and place; the use of multi-user networked projects to give a sense of inhabitation and to enable collaborative design and critique.

In order to place our research in context we first review the legacy of narrative drawing that was prevalent at the Architectural Association London during the 70's and 80's and though this discussion we position an approach to digital visualization that aims to evoke the non objective aspects of architecture. We then describe the software development of a collaborative virtual environment (string CVE) that is based on extensions to a commercial game engine. Outcomes from the use of this software in the context of architectural education are reported and as a consequence to this application testing we describe future work on an integrated project database that will address identified problems.

\section{After projection: Narrative Drawing}

Robin Evans's seminal work 'The Projective Cast' [2] is a history of architecture with a difference - it traces the impact of drawing technique and makes the argument that architecture has been constrained and in turn liberated by developments in the ability to describe form via projective geometry. Plan, elevation, section and perspective line drawing have been the primary tools of visualization for generations of architects and according to Evan's thesis, 
drawing technique have had a direct effect on theory and practice. The development of perspective, the use of ruled surface to approximate curvilinear geometry, and the objectifying use of parallel projection to see the buildings in its entirety are compelling examples that Evans uses to discuss the impact of technique and its assimilation with ways of thinking about architecture.

For modernist architects of the last century who embraced the rallying call of a 'machine for living' architecture was conceived as a neutral mechanism articulated via plan extrusions that neatly organized structure and cladding into functional zones and circulation systems. Of all the projections the plan was seen as 'the generator' with the most 'truthful' visualizations being parallel projections that literally presented buildings as dimensioned objects.

By the late 1970's and 80 's there was a re-evaluation of the role of the drawing particularly at the Architectural Association London where Bernard Tschumi reasserted the importance of the functional program for architecture. Inspired by a situationist view of the city Tschumi's continuing aim is to re assert the importance of architecture as place for the unfolding of events. A key strategy in this aim was experimentation with alternate forms of architectural visualization and his design classes at the architectural association were among the first to attempt to inform architectural drawing with a sense of occupation through time. Drawings were annotated with evocative texts and photography was obsessively used "as live insert, as artificial documentation, as a hint of reality interposed in architectural drawing" [3].
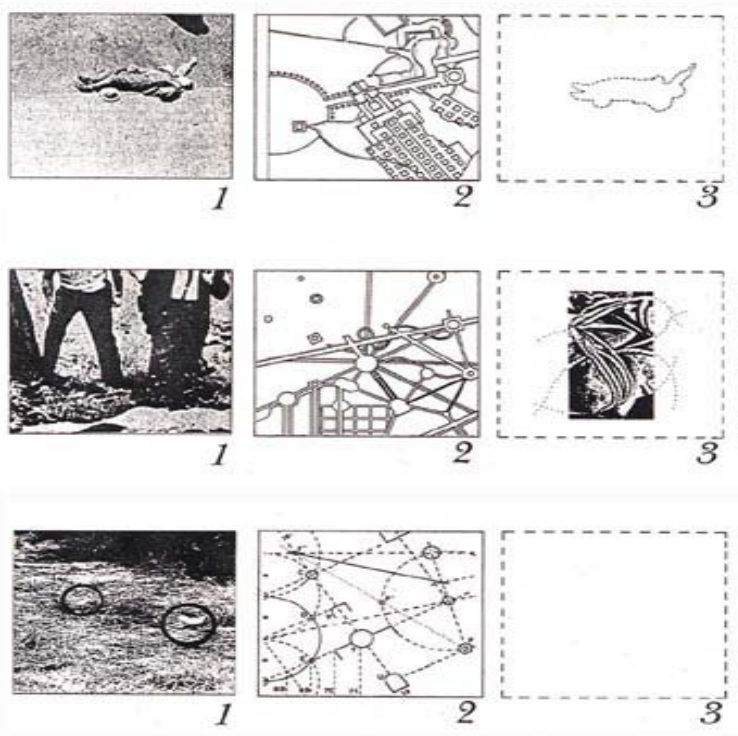

Figure 1. Bernard Tschumi, Manhattan Transcripts.
Bernard Tschumi's Manhattan Transcripts of the 1980s employed a graphical notation system that mixed events, spaces, and motion [4]. The term 'narrative drawing' was coined as a term to describe this inventive research that explored the relationship between functional programme and building and this innovative approach continued after Tshcumi through a former student Nigel Coates.

"It was said from the beginning that architecture lay somewhere between the body and the city, that it was by no means exclusive, but dependent on the experiences it contained. It therefore had to consider fleeting meanings as well as solid architectural forms, movement as well as the volumes that contained them, the subjective as well as the rational - in fact, be an interpenetration of structures and life itself" [5].

The mode of design visualization for this 'interpenetration' was narrative drawings: lines produced via the drawing board montaged with text, time notation, and photographs; all artfully rendered in graphite and charcoal. They represent a fascinating exploration of architectural visualisation that is of interest to our research into the potential use of virtual environments in the context of design education. One interpretation of the wholesale take up of digital drawing or modelling within architecture that has occurred over the last ten years is that there has been a regression in terms of the interpenetration of object, place and the event (certainly in relation to narrative drawing) and a reinforcement of the paradigm by which architecture is conceptually and in many cases literally developed as machine like object. Architecture can now be modelled to the last rivet, rendered seductively in burnished aluminium, and take form via the machines of computer aided manufacture (CAM). This process is compelling and we have argued previously that this allows the re introduction of a sense of craft that short circuits Robin Evan's thesis via the use of parametric digital models linked directly to CAM machines [6].

The advantages of working at this higher level where associative geometry and generative approaches to form allow a high degree of refinement are clear in terms of the creation of unique geometry. Our concern is that concentration on the properties of the object within the contextual vacume of this advanced software negates an approach to architecture as place for the unfolding of events, particularly in the context of design education. This view does not undermine the value of these advanced process but ideally allows their incorporation within a virtual environment to allow a sense of occupation and context, particularly during the important early stages of design. 


\section{String CVE software development}

Architecture was seen as one of the logical benefactors of virtual reality technologies in terms of design visualisation but in practice the cost of software and hardware has resulted in minimal impact. The School of Architecture at the University of Auckland and SIAL has been experimenting with multiplayer games software as a feature-rich but low cost alternative to high-end virtual reality software. While the applications themselves are not appropriate for education usage, the underlying engines are graphically sophisticated, offer advanced multi-user network capability and are designed to perform well on standard hardware and operating systems. Given these advantages and the comparative high cost of commercial virtual reality (VR) systems there has been some interest in the use of game engines for a variety of architectural applications [7]. Most of this activity has involved using the content editing software distributed with such games as HalfLife and Unreal Tournament. Our early experiments with Halflife proved successful but also revealed some limitations and by 2001 it was decided to investigate options for developing a more educationally orientated application. The key factors in the choice of a development platform besides good graphic and sound capability were support for large scale environments, robust multi-user capability on low band width, and of course access to engine source code to allow new functionality to be developed. Access to source code is usually prohibitive because of cost but in June 2001 Garage Games released the Torque game engine SDK [8] at \$US 100 per license and in late 2001 we started development. Named 'String CVE' the initial focus of the software development is on producing functionality that allows exploration of 'place for the unfolding of events' as introduced above.

Deliberately positioned as an application that compliments high end CAD software our approach is tailored towards use in the context of architectural education where design teaching is studio based. A teaching studio in architectural education is a mode of teaching by 'the project'. The aim is to encourage creative thinking and conceptual design skills and as a result the outcomes are usually sketch designs that communicate design concepts rather then projects resolved to a high level of detail. In this context the restrictions of game engines in terms of geometry and level of detail are less important and similar to the approach of narrative drawing our emphasis is on evoking the experience of place over time. Game engines are not capable (yet) of producing detailed geometry, photorealistic lighting or acoustically correct sound in real time. We accept these limitations and in turn stress the evocation of context via image and sound, the use of real time computation to allow a sense of immediacy when designing, and the value of collaborative working made possible by extending the multi user network functionality.

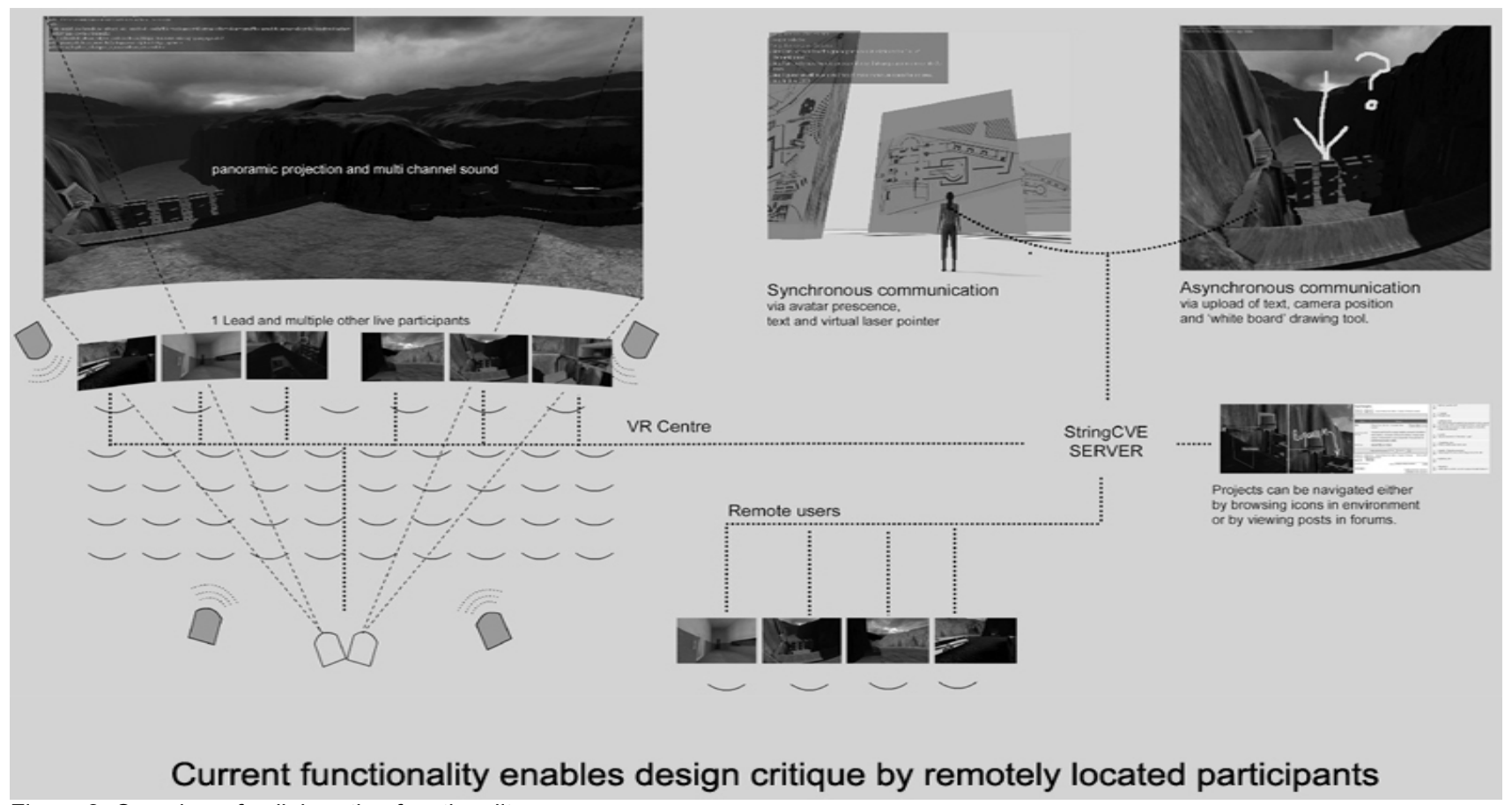

Figure 2. Overview of collaboration functionality 


\subsection{Asynchronous Collaboration}

Our primary development to date has been in the extension of network capabilities to allow asynchronous collaboration. The core torque engine allows real time communication as usually found in multi user game engines: any client application can operate as a server and up to 128 users can share an environment simultaneously; users are represented as avatars and the location and avatar triggered events (pre recorded animations) are continually updated on each client; while an embedded text chat box allows remote users to share and interact in the same environment. Our first enhancements evolved writing a parsing program to automate the upload and downloading of subsequent projects. Working with a game engine involves handling multiple types of data and the management of this proved difficult for design orientated students. Our interface reduces the need for the user to manage design content - once the publish project option is chosen all the data is copied to a folder and uploaded to the server. Other users could then see the newly published project and in a reverse project the data can be downloaded into the required directories and immediately accessed. We subsequently developed an asynchronous communication module to facilitate internet-based design critique with users in different time zones. This functionality has been implemented using a mySQL database linked to a PHP chat forum to enable 3D browsing of comments and 'white board' type mark ups of the designs. Icons are placed within the 3D scene and a mouse click will 'teleport' the user to the camera position of the author and simultaneously overlay any annotation and open up the text forum. Alternatively projects can be browsed by locating new posts in the forum thus teleporting the user to locations within the 3D world.

\subsection{Sound}

The core torque engine utilises openAL to provide 2D and 3D sound. We have implemented an alternative to this using the Aureal SDK [9] to implement multi channel sound output to enable surround sound.

\section{Software Trials}

We present below some observations of the software in use for two architectural design studios - Memory games and Future Sounds - conducted by the authors at UoA and SIAL. The Memory Games studio in 2002 (with SIAL staff Mark Burry and Gregory More) was undertaken prior to the development of the asynchronous design functionality and was an initial test of the real time environment to allow better understanding of spatial sequence and the possibilities for the use of sound to evoke materiality and context. The brief proposed a central theme of memory and its role in the collective and individual experience of the city. The pedagogical aim is that students understand how the built environment resonates with the sounds of human presence, natural events and technology. The sound samples are not acoustically correct but are used primarily as a form of notation - in effect a 3D 'sound sketch'. Sound was used to communicate intent in terms of spatial characteristics or materiality and as importantly the pre recorded sound conveyed a sense of occupation and functional use. Sounds from café, street or private spaces were arranged in conjunction with visuals to stimulate discussion over appropriate usage and to indicate performance over a 24 hour or seasonal cycle. At the end of the studio, the individual works resided in a series of collective spaces. These spaces contained varied readings of Melbourne's CBD. Two approaches are illustrated below.

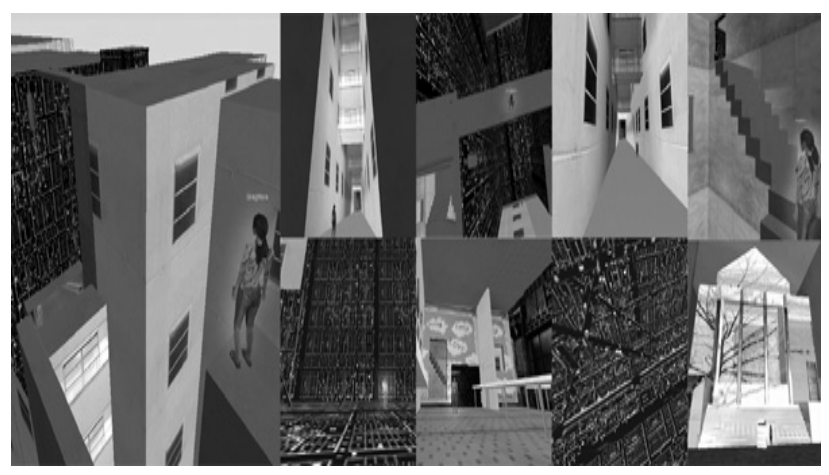

Figure 3: Student project by Laura Harper.

- The work of Laura Harper recaptured the events from her chosen site, which at certain times of day becomes an urban 'soup kitchen'. Her environments merge parallel 'mirrored' spaces which give a stimulating sense of déjà vu.

- The work of Fooch Chi examined a graphical or iconographical representation of the CBD. He defined place by sound, allowing the city to be 'reread' literally as textual entities and sound.

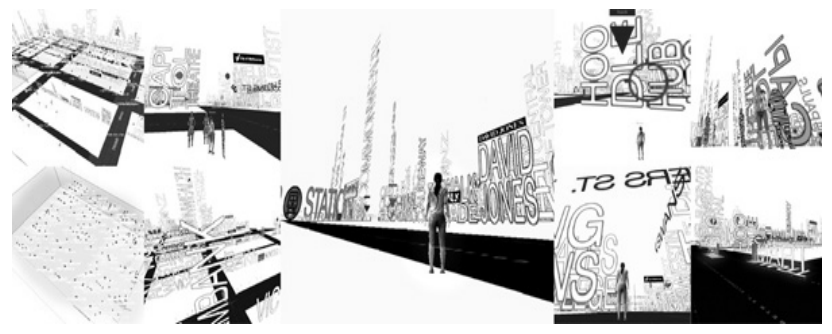

Figure 4: Student project by Fooch Chi. 
In summary the memory games studio demonstrated the following points in relation to the effectiveness of the software to meet the research aims.

- The use of the upload / download functionality made it possible to effectively share projects amongst distributed users. Students and tutors would schedule shared sessions where design evaluation could occur via avatar interaction and the use of text chat.

- Sound was a more effective way of communicating functional use then graphics. However the quantity and quality of the audio files created more substantial file sizes, limiting the distribution potentials of the CVE.

- The ability to explore projects in real time demonstrated the importance of context and challenged the 'object' based thinking of typical design students. The importance of spatial sequence was emphasized.

In the second studio, The Future Sound of Cities the asynchronous communication module was implemented. The studio would continue the focus on sound augmented visualization and also test the effectiveness of the software to support collaboration between students in Auckland, Melbourne and a tutor located in London. In general this studio confirmed the results of the first studio but the asynchronous communication module was not successful due to the following factors.

- Poor interface design. There was a marked difference in use between the Auckland students who worked together in a university studio with constant access to equipment and technical help. These students managed to work together in small groups on the same project and regularly upload work for critique. In contrast the Melbourne students who had limited resources and worked primarily from home did not collaborate successfully with each other or upload projects regularly. This highlighed the difficulty of creating game engine compatable graphics and sound and that the working environment and asynchronous communication interface were not sufficiently intuitive, but also underlined the importance of the social aspects of studio culture in facilitating group dynamics and the sharing of technical skill.

- $\quad$ Lack of immediacy. There would usually be a 1 to 2 day time difference between projects being uploaded and the review of the project. Often by the time dialogue had been established the students had made design changes that could not immediately be shared.
- Media focus. Perhaps due to the success of the first studio there was too much emphasis on sound with a corresponding lack of graphical sophistication. Some students became disillusioned with the technical skill required to produce sophisticated sound environments.

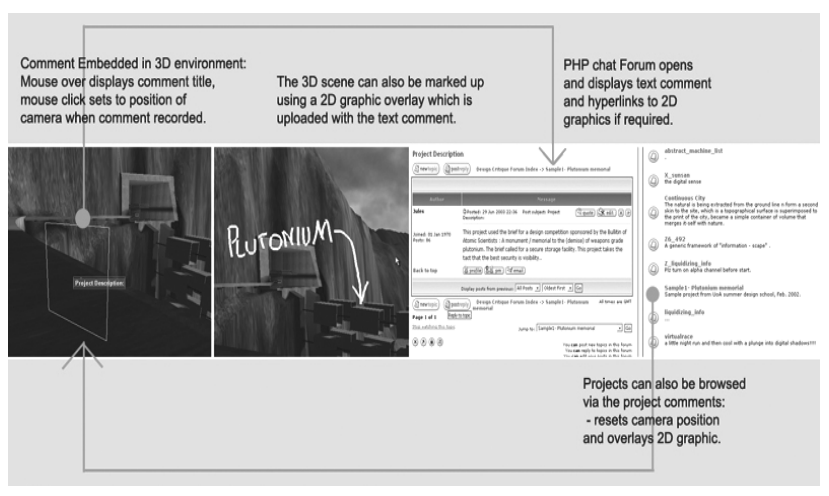

Figure 5 : Current Asynchronous communication functionality.

\section{Subsequent Software development.}

There are factors beyond the scope of the software development that affected the success and failures of the above design studios. The dynamics of different groups of students and the role of design studio culture - students and tutors conversing face to face - are important factors. There are however two clear outcomes: the value of using a virtual environment to allow an understanding of design context and in particular the important potential of sound to augment visualisation; and the need to rethink functionality to support collaborative design and critique.

Consequently we are examining options for better sound functionality and have developed an approach to collaboration that uses an integrated project database to allow projects to be dynamically edited and viewed by multiple users. In anticipation of adapting more advanced games engines the database is been designed to allow data to be exported and imported from standard CAD software. (The importance of data transfer has been discussed in a recent paper in which we position the use of a CVE in relation to CAD [10].) Implementation of the integrated project database is underway and we hope to present an alpha version at the conference which will have the following features.

- Allow multiple users to access a database server and add or modify design components. On client login new design components and design edits are detected and the client updated.

- Allow any client to manipulate the design (environment and components) and propagate 
these updates to multiple clients in real time.

- $\quad$ Provide managed control of the data set: levels of access are to be assigned to each user and design; implementation of 'save edit' and 'design history' functions are planned with each edit able to be undone (i.e. users can step through design versions).

- Extend existing asynchronous design critique functionality to allow comments to be recorded with each design iteration.

\section{Conclusion and further work}

The use of a game engine based collaborative virtual environment has enabled the development of software to support architectural design education. The focus of this paper has been on establishing the role of a CVE to allow an exploration of design context in a manner that is not possible when using typical architectural visualization software. The advantages of working in a real time environment where early design iterations can be tested from multiple points of view, and the value of using sound to evoke occupancy and materiality, allow students to understand architecture as place for the framing of events over time. In the context of design education where there are declining resources to support traditional studio teaching there is a need for software that allows distance collaboration. The focus of our next design studio will build on our previous outcomes and test the integrated project database functionality to support design collaboration between remote users and tutors.

We are also interested in the capacity for a CVE to augment the visual and the aural with information that gives the designer real time feedback on design attributes such as floor area and environmental performance. This embedded information need not be highly accurate for the early stages of design but provide approximate values on building performance to enable the designer to monitor the implications of different basic approaches. A precedent for this development is the Pangea software developed by researchers at the Bartlett School of Architecture that linked computer models to a range of techniques (Neural Nets, Genetic Algorithms, Fuzzy Logic) that communicate design performance to the designer [11]. We plan to develop similar performance based modules in future work and to this end we are currently evaluating an alternate engine that uses LISP as a scripting language.

\section{References}

[1] J. Thackara. Design After Modernism. London, Thames and Hudson. 1988. p. 26

[2] R. Evans. The Projective Cast: Architecture and its Three Geometries. Cambridge, Mass. MIT Press. 1995.

[3] B. Tschumi. Spaces and Events. In Exhibition Catalogue The Discourse of Events. London. Architectural Association. 1983. p.8

[4] B. Tschumi. The Manhattan Transcripts. London.Academy Editions. 1994.

[5] N. Coates. Narrative Breakup. In Exhibition Catalogue The Discourse of Events. London. Architectural Association. 1983. p.12.

[6] J. Moloney. Digital Craft. Proceedings of the $32^{\text {nd }}$ Annual Conference of the Australia and New Zealand Architectural Science Association", Wellington. 1998. pp $289-29$

[7] M.F. Shiratuddin \& W. Thabet. Virtual Office Walkthrough using a 3D Game Engine. International Journal of Design Computing Vol. 4.url: http://www.arch.usyd.edu.au/kcdc/journal/vol4/

[8] GarageGames url:http://www.garagegames.com

[9] Aureal url:http://members.optushome.com.au/kirben/3ds oftware.html

[10] J. Moloney \& R, Amor. String CVE: Advances In a Game Engine Based Collaborative Virtual Environment For Architectural Design. Proceedings of the International Conference on Construction Applications of Virtual Reality, Blacksburg Virginia U.S.A., 2003, pp.156-168.

[11] Pangea url:http://www.cebe.cf.ac.uk/learning/habitat/HA BITAT2/pangea.html 